\title{
IMMUNOHISTOCHEMICAL DIAGNOSIS AND PROGNOSIS OF SMALL CELL LUNG CANCER: THE SEARCH FOR NEW STRATEGIES
}

\author{
Irina Yakovtsova, Olexandr Yanchevskiy, Taisiia Chertenko, Andriy Kis, \\ Andriy Oliyinyk
}

The aim: to find the optimal combination of immunohistochemical markers for differential diagnosis and prognosis of small cell lung cancer in small biopsy samples.

Materials and methods. The tumor specimens were divided into 3 groups: 1) 25 biopsy samples of small cell lung cancer before treatment; 2) 25 samples of small cell lung cancer procured from autopsies of patients, who underwent chemotherapy; 3) 15 biopsy samples of other lung tumors histologically similar to SCLC. All tumor samples were formalin fixed and paraffin embedded (FFPE). Immunohistochemical study performed with 5 primary antibodies: CD56, pl6ink4A, TTF-1, CD117, Ki-67.

Results. TTF-1 was positive in all small cell lung cancer, lung adenocarcinomas and atypical carcinoids. Expression of CD56 was positive in $100 \%$ of tumors from $1^{\text {st }}$ group and $92 \%$ of these tumors had more than $25 \%$ of positive tumor cells. Expression of pl6ink4A was significantly higher in $1^{\text {st }}$ group than in the $3^{\text {rd }}$ one $(p<0,001)$. The stepwise logistic regression was used for finding the best markers for differential diagnosis of small cell lung cancer in small biopsy samples. The next combination of markers was chosen: TTF-1/CD56 (score 2-4)/p16 ink4A/CD117 (sensitivity - 80 \%; specificity $-86.67 \%$; $<<0,001)$ where "score 2-4" means expression of CD56 more than in $25 \%$ tumor cells. Expression of Ki-67 was higher in the $2^{\text {nd }}$ group compared with the $1^{\text {st }}$ one $(p<0,001)$.

Conclusion. Evaluation of p16 expression can be used as additional marker for differential diagnosis of small cell lung cancer. The following combination of markers: TTF-1/CD56 (score2-4)/p16 ink4A/CD117 could be useful in diagnosis of small cell lung cancer in small biopsy samples and in the choice of targeted chemotherapy. The further study in paired tumor samples of small cell lung cancer before and after chemotherapy is required to prove the significance of changes in expression of Ki-67, CD56, CD117 and p16ink4A

Keywords: small cell lung cancer, small biopsy sample, expression of p16ink4A, CD117, pathomorphosis

How to cite:

Yakovtsova I., Yanchevskiy O., Chertenko T., Kis A., Oliyinyk A. (2022). Immunohistochemical diagnosis and prognosis of small cell lung cancer: the search for new strategies. ScienceRise: Medical Science, 1 (46), 31-36. doi: http://doi.org/10.15587/2519-4798.2022.253047

(C) The Author(s) 2022

This is an open access article under the Creative Commons CC BY license hydrate

\section{Introduction}

Small cell lung cancer (SCLC) accounts for $15 \%$ of all lung cancers in the world [1]. SCLC has many genetic mutations, which on the one hand causes a very good primary response of these tumors to cytotoxic chemotherapy, and on the other hand leads to the rapid development of chemoresistance [2, 3]. Unlike non-small cell lung cancer (NSCLC), no effective treatment has yet been found for SCLC in recent years [3, 4]. The 5-year survival rate for patients is, according to various data, 2 $7 \%[1,2,5,6]$, and for patients in the early stages of SCLC who received combination radiation and chemotherapy up to $34 \%$ [1]. Modern SCLC treatment strategies include chemotherapy before surgery, with rare exceptions when no regional lymph node involvement is detected, so SCLC pathomorphological diagnosis is performed on small biopsy (approximately $75 \%$ of cases) or cytological specimens (approximately $2 \%$ ). [7]. Histological diagnosis of SCLC in small biopsy specimens could sometimes be a challenge for the pathologist, espe- cially in the presence of artifacts and necrosis in the biopsy. Differential diagnosis of SCLC is performed with other neuroendocrine lung tumors (mainly typical and atypical carcinoids), squamous cell lung cancer (especially its basaloid variant), round cell sarcomas (e. g., Ewing's sarcoma), lymphomas, and sometimes with high-grade adenocarcinomas of lungs or other primary localizations. In severe cases, the immunohistochemical method is used for differential diagnosis [1]. In small biopsies, the number of sections is limited, so it is important to choose the optimal panel of markers that not only helps to put an accurate diagnosis, but also provides additional opportunities for tumor prognosis and chemotherapy strategies. One of the markers in the diagnostic panel must be TTF-1, which will confirm the pulmonary origin of the tumor, but it should be noted that carcinoids are often negative for TTF-1 with a positive reaction only at the periphery of the tumor, also negative are squamous cell carcinomas of lungs $[1,8,9]$. It is also mandatory to use at least one of the neuroendocrine markers, such as 
CD56, chromogranin A or synaptophysin [1, 8]. CD 56 is the most sensitive, but the least specific, so its use in the panel for small biopsies may be a reasonable choice [1]. Thus, in one of the scientific papers it is recommended to use a combination of three markers TTF-1 + CD56 + $+\mathrm{p} 16$ ink4A for differential diagnosis of SCLC. Positive expression of p16ink4A in $94 \%$ SCLC was observed, and the combination with CD56 allowed to clearly distinguish SCLC from NSCLC [10]. Ki-67 may also be a useful marker in the differential diagnosis between carcinoids and SCLC. Proliferation index (PI) above $30 \%$ in the vast majority of cases indicates in favour of SCLC, but sometimes errors are possible in cases of atypical carcinoids [1,9]. Another interesting additional marker, both for differential diagnosis and for the choice of targeted therapy may be CD117 [9, 11, 12]. According to various data, from 60 to $80 \%$ of SCLC could express CD117 [9, 11], which could theoretically be used to prescribe tyrosine kinase inhibitors.

The aim of the research. To optimize the marker panel for SCLC in small biopsy specimens, which is needed to improve differential diagnosis, predict tumor progression, select individual chemotherapy regimens, and distinguish between SCLC cases for chemotherapy and chemoresistance cases.

\section{Materials and methods of the research}

Three groups were formed for the study: the first group included 25 cases of SCLC biopsies before treatment, the second group included 25 cases of autopsy SCLC after chemotherapy, and the third group included 15 cases of biopsies of anaplastic forms of lung cancer and carcinoids histologically similar to SCLC. Biopsies of SCLC, NSCLC and carcinoids were selected at the "National Cancer Institute" of the Ministry of Health of Ukraine in Kyiv and the "Prime Test" laboratory in Kharkiv for the period 2010-2020, SCLC autopsy material was selected at the Department of Pathology Municipal non-profit enterprise "City Multidisciplinary Clinical Hospital No. 17" of Kharkiv City Council for the period 2015-2020.

The design of the study and all the methods used in the study were approved by the Commission on Bioethics of the "Kharkiv Medical Academy of Postgraduate Education" (minutes No. 2, dated 14.09.2021) and meet the requirements of the Declaration of Helsinki. There are existing agreements on scientific and practical cooperation with the institutions where the material was selected.

From medical histories and autopsy protocols were obtained following information: sex, age of patients, treatment (surgery and chemotherapy). All selected material was represented by specimens of tumors fixed in formalin and embedded in paraffin blocks. Inclusion criteria were complete clinical information about the patient, as well as information on further treatment or received treatment in autopsy cases, sufficient material for immunohistochemical study, and the appropriate quality of the material.
The following panel of markers was used for immunohistochemical study: CD56 (monoclonal antibody, clone 123C3, Dako, 1:100), p16ink4A (monoclonal antibody, clone 1D7D2, ThermoScientific, 1:200), TTF-1 (polyclonal antibody20, PA5- ThermoScientific, 1:500), CD117 (polyclonal antibody A4502, Dako, 1:250), Ki-67 (monoclonal antibody, clone SP6, ThermoScientific, 1:400). All slides with immunohistochemical sections were evaluated by two pathologists using a Primo Star microscope (Carl Zeiss), x4, x10, x40, x100 lenses, for microphotos AxioCam ERc5s camera was used.

The expression of markers such as TTF-1, CD56, p16ink4A was evaluated on a semi-quantitative scale by the number of positive cells in the tumor, where 0 points corresponded to the expression level $<5 \%$ of tumor cells, 1 point expression level from $5 \%$ to $25 \%, 2$ points expression level 26-50 \%, 3 points $-51-75 \%$ ) and 4 points $->75 \%$ [10].

The proliferation index (PI) for Ki-67 was calculated as the ratio of immunopositive tumor nuclei to the total number of tumor cell nuclei without considering the intensity of staining. The count was performed in three areas with the most pronounced immunohistochemical response ("hot spots") at X400 magnification.

Membrane and cytoplasmic expression of CD117 was assessed by a semiquantitative method, where $0-$ no staining or less than $1 \%$ of tumors; $1+$ focal expression, or low color intensity up to $60 \%$ of tumor cells; $2+-$ weak color in more than $60 \%$ of tumor cells, or moderate intensity in 30-79\% of tumor cells, or expressive intensity in less than $30 \%$ of tumor cells; $3+-$ moderate intensity of staining in more than $80 \%$ of tumor cells, or expressive staining in more than $30 \%$ of tumor cells [13].

The level of agreement between the two pathologists regarding the percentage of marker expression was assessed using the interclass correlation coefficient (ICC) calculated in MedCalc (Version 20.009, trial).

Fisher's exact test was used to assess the association between traits. Differences between quantitative indicators, namely PI on Ki-67 in SCLC groups before treatment and autopsy cases of SCLC after PCT were assessed using the non-parametric Mann-Whitney test. Extensive indicator (\%) was also calculated for qualitative description. Where possible, the median was calculated, which better than the arithmetic mean describes the sample. Step-by-step logistic regression with ROC analysis and ROC curve construction was also performed. The primary tables for the calculation were generated in $\mathrm{Mi}$ crosoft Excel 2013 with subsequent statistical processing in MedCalc (Version 20.009, trial).

\section{Research results}

The median age of patients was 60 years for primary SCLC, 66 years for deaths from SCLC after chemotherapy, and 57 years for all other tumors. The vast majority of patients in our groups were men. More detailed information is presented in Table 1 . 
Age and sex characteristics of patients in the study groups

\begin{tabular}{|c|c|c|c|}
\hline $\begin{array}{ll}\text { Characteristics } & \text { Groups } \\
\end{array}$ & $\begin{array}{c}\text { SCLC } \\
\text { (Group 1), N=25 }\end{array}$ & $\begin{array}{c}\text { SCLC } \\
\text { (Group 2), N=25 }\end{array}$ & $\begin{array}{l}\text { Other lung tumors } \\
\text { (Group 3), } \mathrm{N}=15\end{array}$ \\
\hline $\begin{array}{l}\text { Age } \\
\text { Median } \\
\text { Range }\end{array}$ & $\begin{array}{c}60 \\
50-74\end{array}$ & $\begin{array}{c}66 \\
54-76\end{array}$ & $\begin{array}{c}57 \\
17-86\end{array}$ \\
\hline $\begin{array}{l}\text { Sex } \\
\text { Male } \\
\text { Female }\end{array}$ & $\begin{array}{c}23 \\
2\end{array}$ & $\begin{array}{c}18 \\
7\end{array}$ & $\begin{array}{c}11 \\
4\end{array}$ \\
\hline
\end{tabular}

The agreement between the two pathologists was very high for each of the evaluated immunohistochemical markers, confirming high levels of intraclass correlation coefficient (ICC): TTF-1- $0.92\left(\mathrm{CI}_{95}: 0.87-0.95, \mathrm{~N}=65\right)$; CD56 - $0.96\left(\mathrm{CI}_{95}: 0.93-0.97, \mathrm{~N}=65\right)$; Ki 67- $0.99\left(\mathrm{CI}_{95}\right.$ : 0.9858-0.9948, N=65); p16-0.97 ( $\left.\mathrm{CI}_{95}: 0.95-0.98, \mathrm{~N}=65\right)$; $\mathrm{CD} 117-0.99\left(\mathrm{CI}_{95}: 0,9850-0.9944, \mathrm{~N}=65\right)$.
In a $100 \%$ SCLC study, TTF-1 was expressed in both biopsies and autopsies. Among the group of histologically similar tumors to SCLC, TTF-1 expression was absent in squamous cell lung cancers, some lung carcinoids, and Ewing's sarcoma. Details of expression levels are shown in Table 2.

Table 2

Expression of TTF-1, CD56, p16, Ki-67 and CD117 in the two study groups SCLC and other lung tumors (data are presented in the absolute number of cases $\mathrm{N}$ )

\begin{tabular}{|c|c|c|c|}
\hline $\begin{array}{ll}\text { Marker, } \\
\text { expression level }\end{array}$ & $\begin{array}{l}\text { SCLC (Group 1) } \\
\qquad \mathrm{N}=25\end{array}$ & $\begin{array}{l}\text { SCLC (Group 2) } \\
\qquad \mathrm{N}=25\end{array}$ & $\begin{array}{l}\text { Other lung tumors (Group 3) } \\
\qquad \mathrm{N}=15\end{array}$ \\
\hline \multicolumn{4}{|c|}{ TTF -1 } \\
\hline 0 & 0 & 0 & 6 \\
\hline 1 & 0 & 1 & 5 \\
\hline 2 & 2 & 12 & 2 \\
\hline 3 & 23 & 12 & 2 \\
\hline 4 & 0 & 0 & 0 \\
\hline \multicolumn{4}{|c|}{ CD56 } \\
\hline 0 & 0 & 0 & 6 \\
\hline 1 & 2 & 3 & 0 \\
\hline 2 & 19 & 18 & 1 \\
\hline 3 & 4 & 4 & 5 \\
\hline 4 & 0 & 0 & 3 \\
\hline \multicolumn{4}{|c|}{ P16 } \\
\hline 0 & 0 & 3 & 7 \\
\hline 1 & 7 & 9 & 7 \\
\hline 2 & 9 & 8 & 1 \\
\hline 3 & 5 & 5 & 0 \\
\hline 4 & 4 & 0 & 0 \\
\hline \multicolumn{4}{|c|}{ Ki-67 } \\
\hline Median & $58 \%$ & $35 \%$ & $20 \%$ \\
\hline Range & $31-73 \%$ & $18-77 \%$ & $0-64 \%$ \\
\hline \multicolumn{4}{|c|}{ CD117 } \\
\hline 0 & 5 & 9 & 12 \\
\hline 1 & 4 & 6 & 1 \\
\hline 2 & 9 & 6 & 2 \\
\hline 3 & 6 & 4 & 0 \\
\hline
\end{tabular}

CD56 was positive in $100 \%$ of SCLC biopsies, with $92 \%$ of the marker being expressed in more than $25 \%$ of tumor cells (expression levels 2-4). In addition, CD56 was expressed in all carcinoids where the expression level was 2 points and above. Significantly significant differences in CD56 expression in SCLC biopsies before chemotherapy and in SCLC autopsy material after chemistry were not found ( $\mathrm{P}$ value of Fisher's exact test $=0.674$ ).
The median $\mathrm{Ki}-67$ for SCLC in biopsies is $58 \%$ (31-73\%), for other tumors - $20 \%(0-64 \%)$. If we look at the level of Ki-67 expression in malignant tumors histologically similar to SCLC, we could see that it is greater than $30 \%$ (Tab. 2), so the use of Ki-67 as a marker for differential diagnosis can be limited to carcinoids. When comparing Ki-67 SCLC expression levels in biopsies and autopsies, PI was higher in biopsy cases 
(before chemistry) than in autopsy cases (58\% and $35 \%$, respectively). The difference in Ki-67 expression level between these groups was statistically significant (MannWhitney U-test $=159, \mathrm{p}=0.0029$ ).

Proportionally, $100 \%$ of primary SCLC tumors expressed p16ink4A (Fig. 1), and $72 \%$ were positively expressed in more than $25 \%$ of tumor cells (2-4 points). In the group of tumors that were not SCLC positive expression of p16 ink4A was observed in $53 \%$, and the expression of p16 (2-4 points) was only $6.7 \%$. The difference in p16ink4A expression between SCLC and other tumors is statistically significant $(\mathrm{P}$ value of Fisher's exact test $=0.00035)$. When comparing the expression of p16ink4A (2-4) in the biopsy group before chemotherapy and in the autopsy group after chemistry, the expression level was higher in the first group (72\% and $52 \%$, respectively), but this difference was not statistically significant ( $\mathrm{P}$ value of Fisher's exact test $=0.24$ ).

CD117 was positive in $80 \%$ of SCLCs (Fig. 2) and in $20 \%$ of other tumors, one of which was Ewing's sarcoma and 2 carcinoids (Tab. 2). CD117 was higher in SCLC biopsies than in autopsy cases $(80 \%$ and $64 \%$, respectively), but this difference was not statistically significant ( $\mathrm{P}$ value of Fisher's exact test $=0.156$ ).

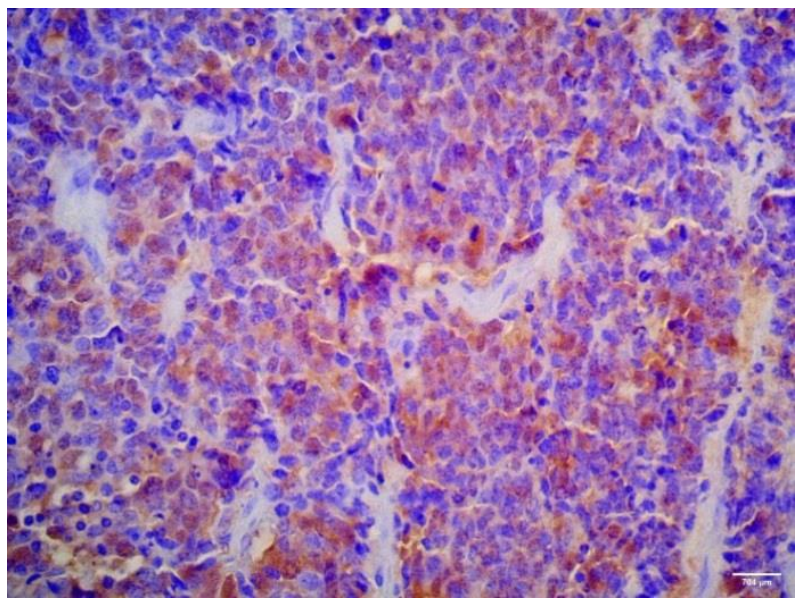

Fig. 1. Expression of p16ink4A in SCLC (3 points), additional staining with Mayer's hematoxylin $(\times 400)$

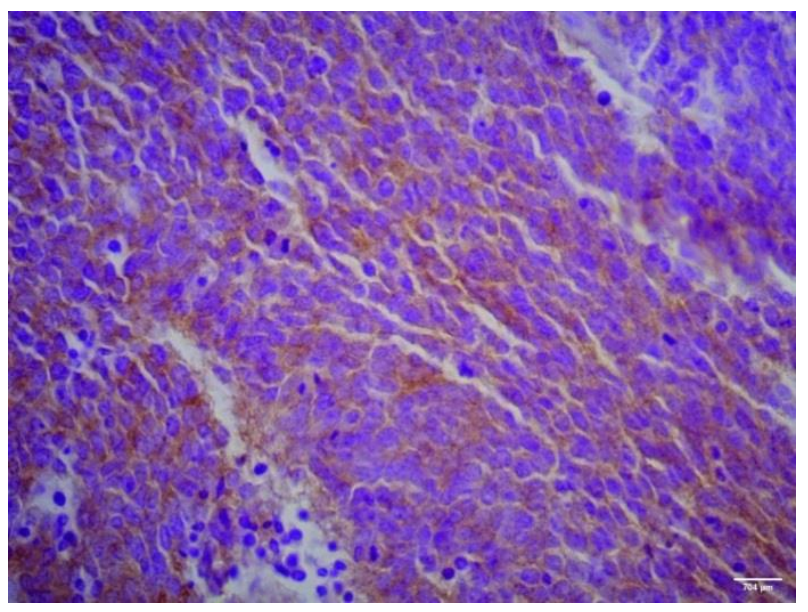

Fig. 2. Expression of CD117 in SCLC (2+), additional staining with hematoxylin Mayer $(\times 400)$

To identify a more efficient set of markers for the differential diagnosis of SCLC in small biopsies, a step-by- step logistic regression was performed that included all study markers and their various combinations. The combination of the following markers proved to be the most effective in the calculations: TTF-1 / CD56 (2-4) / p16 ink4A / CD117 (sensitivity - $80.0 \%$, specificity $-86.67 \%$; $\mathrm{p}=0.0003$ ). That is, the expression of TTF-1 $5 \%$ and more positive tumor cells, the expression of CD56 - 26\% and more positive tumor cells, the expression of p16ink $4 \mathrm{~A} \geq 5 \%$ and the expression of CD117 1+ and more. ROC analysis was also performed with the construction of the ROC curve. Area under the ROC curve (AUC) $=0.833$ (CI95: 0.6820.93; $\mathrm{S}=0.0611$; $\mathrm{z}$-score=5.458; Youden index=0.6667).

\section{Discussion}

In our study, we used both traditional markers for the differential diagnosis of SCLC such as TTF-1, CD56 and $\mathrm{Ki}-67$, and markers that in addition to diagnostic value could also be used to predict and select new schemes of targeted chemotherapy: p16ink4A and CD117. Our data on the expression of TTF-1 and CD56 did not contradict the data of the latest histological classification of WHO thoracic tumors [1]. PI Ki-67 was also higher in SCLC than in typical carcinoids and even atypical carcinoids, which is consistent with world data [1, 9, 14], but Ki-67 expression levels in other malignancies other than SCLC did not differ significantly from SCLC (Tab. 2). In our study, we obtained data on a statistically significant difference in p16ink4A expression between SCLC and other tumors ( $\mathrm{P}$ value of Fisher's exact test=0.00035). The our data is similar to the data of a group of scientists who observed significantly higher expression of p16ink4A in SCLC (95\%) compared with squamous cell lung cancer $(50 \%)$ and lung adenocarcinoma $(41 \%)$ [10]. Because p16ink4A is not a specific marker for SCLC, but a marker that characterizes cell cycle disruption by loss of a specific antiapoptotic factor, retinoblastoma protein (pRB) [15], it could be used for the differential diagnosis of SCLC only in combination with other antibodies, for example TTF1/CD56/p16ink4A, as suggested by M. Svajdler and coauthors [10], or in the combination obtained by stepwise logistic regression in our study: TTF-1/CD56 (2-4)/p16ink4A/CD117 (sensitivity - $80.0 \%$, specificity $86.67 \%$; $=0.0003$ ). The combination we obtained was more effective than the use of markers alone, or any other combination of markers we studied, in particular combinations with Ki-67, combinations that did not take into account how pronounced was the expression of CD56, and combinations without CD117. The decision on the possible inclusion of CD117 in the diagnostic scheme was based on the data of the WHO histological classification in 2015, which indicated that CD117 may be important in the differential diagnosis of SCLC, as it is expressed in more than $60 \%$ of these tumors [9], data from our study, where we saw that CD117 expression was observed in $80 \%$ of SCLC. These data also coincide with the data of Pelosi et al., according to which CD117 expression was observed in 67-80\% of SCLC, but only in $7 \%$ of carcinoids (however, it is not specified whether they were typical or atypical carcinoids) $[11,16]$. We did not detect the expression of CD117 in squamous cell lung cancer and lung adenocarcinomas, although according to Vidya Jha et al., it could be observed in $70.17 \%$ of 
adenocarcinomas and $23.4 \%$ of squamous cell lung cancers [17], which also indicates that CD117 is not a specific biomarker for SCLC. This difference in CD117 expression between our NSCLC cases and other studies is primarily due to the small number of adenocarcinoma and squamous cell carcinomas in our sample (2 and 3, respectively), but the presence of CD117 expression in Ewing's sarcoma in our sample is not consistent with world data [18]. However, it should be noted that the use of CD117 may be less diagnostic than prognostic and may be important for the selection of candidates for specific targeted therapy with tyrosine kinase inhibitors. Studies of SCLC cell lines expressing the c-Kit receptor (CD117) have revealed the sensitivity of these cells to targeted therapy with c-Kit and c-Met inhibitors (imatinib and crizotinib) [12].

When comparing autopsy cases of SCLC after polychemotherapy and cases of primary SCLC in biopsy material, from patients who had not yet been treated, we noted that although the percentage expression of CD56, CD117 and p16ink4A was lower in cases after PCT, no statistically significant difference between groups was. The only statistically significant difference was observed in the expression of $\mathrm{Ki}-67$, which was lower in the group after PCT (Mann-Whitney U-test=159, p=0.0029). N. Ishibashi and co-authors point out in their paper that patients with SCLC with lower Ki-67 respond less well to radiotherapy, which in some cases has been combined with PCT and have poorer survival [19], which is partly consistent with our data because all our cases of patients after treatment are autopsies. However, on the other hand, a lower PI for Ki-67 in the SCLC autopsy group may be a consequence of the treatment. To confirm or refute the hypothesis of the prognostic significance of Ki-67 expression, paired cases of SCLC from patients before and after radiotherapy or PCT are required.

Study limitations. To clarify the results, it is necessary to study the expression of CD56, CD117, p16ink4A and Ki-67 in large groups of patients with both SCLC and NSCLC. Paired cases of SCLC before and after treatment are needed to better understand changes in the expression of CD56, CD117, p16ink4A and Ki-67 markers.

Prospects for further research. Determination of CD117 and p16ink4A expression may be useful in the future both for a better understanding of SCLC oncogenesis and for the selection of individualized chemotherapy for patients.

\section{Conclusions}

1. Expression of p16ink4A is significantly higher in SCLC than in other lung tumors histologically similar to SCLC (P value of Fisher's exact test $=0.00035$ )

2. For the differential diagnosis of SCLC in small biopsy specimens, we recommend the following combination of immunohistochemical markers: TTF-1 / CD56 (2-4 points) / p16 / CD117 (sensitivity $-80.0 \%$, specificity $-86.67 \% ; p=0.0003$ ), where the term " $2-4$ points" means the presence of positive expression of CD56 in more than $25 \%$ of tumor cells. The proposed combination of markers may also be useful in the future for the selection of targeted therapy for patients with SCLC.

3. Ki-67 expression is significantly lower in the SCLC autopsy group after PCT than in the pre-treatment SCLC biopsy group (Mann-Whitney U-test=159, $\mathrm{p}=0.0029)$, but SCLC studies are needed to confirm the significance of this feature, before and after treatment.

\section{Conflict of interests}

The authors declare there is no conflict of interests.

\section{Funding}

The work was performed within the research No. 0117U000594: "Pathohistological and immunohistochemical diagnosis and prognosis of malignant tumors of different localization, taking into account their biological properties and clinical course".

\section{Acknowledgments}

The authors are grateful to the pathology department of the "National Cancer Institute" of Kyiv for providing histological material to small cell lung cancers and other lung tumors histologically similar to small cell lung cancers. We are also grateful to the archives of this institution for accessing patients' medical histories and all information regarding their treatment. We are also grateful to the pathology department of "CCMH-17" for the opportunity to select autopsy material from patients with SCLC and access to autopsy protocols.

\section{References}

1. Lokuhetty, D. (2021). Thoracic tumours. WHO Classification of Tumours Editorial Board. Vol. 5. Lyon: International Agency for Research on Cancer. Available at: https://publications.iarc.fr/595

2. Raso, M. G., Bota-Rabassedas, N., Wistuba, I. I. (2021). Pathology and Classification of SCLC. Cancers, 13 (4), 820. doi: http://doi.org/10.3390/cancers13040820

3. Schulze, A. B., Evers, G., Kerkhoff, A., Mohr, M., Schliemann, C., Berdel, W. E., Schmidt, L. H. (2019). Future Options of Molecular-Targeted Therapy in Small Cell Lung Cancer. Cancers, 11 (5), 690. doi: http://doi.org/10.3390/cancers11050690

4. Nicholson, A. G., Chansky, K., Crowley, J., Beyruti, R., Kubota, K., Turrisi, A. et. al. (2016). The International Association for the Study of Lung Cancer Lung Cancer Staging Project: Proposals for the Revision of the Clinical and Pathologic Staging of Small Cell Lung Cancer in the Forthcoming Eighth Edition of the TNM Classification for Lung Cancer. Journal of Thoracic Oncology, 11 (3), 300-311. doi: http://doi.org/10.1016/j.jtho.2015.10.008

5. Yang, S., Zhang, Z., Wang, Q. (2019). Emerging therapies for small cell lung cancer. Journal of Hematology \& Oncology, 12 (1). doi: http://doi.org/10.1186/s13045-019-0736-3

6. Bunn, P. A., Minna, J. D., Augustyn, A., Gazdar, A. F., Ouadah, Y., Krasnow, M. A. et. al. (2016). Small Cell Lung Cancer: Can Recent Advances in Biology and Molecular Biology Be Translated into Improved Outcomes? Journal of Thoracic Oncology, 11 (4), 453-474. doi: http://doi.org/10.1016/j.jtho.2016.01.012

7. Koinis, F., Kotsakis, A., Georgoulias, V. (2016). Small cell lung cancer (SCLC): no treatment advances in recent years. Transl Lung Cancer Res, 5 (1), 39-50. doi: http://doi.org/10.3978/j.issn.2218-6751.2016.01.03

8. Leslie, K., Wick, M. (Eds.) (2018). Practical Pulmonary Pathology: A Diagnostic Approach. Elsevier, 811. doi: http://doi.org/10.1016/c2015-0-01043-7 
9. Travis, W. (2015). WHO classification of tumours of the lung, pleura, thymus and heart. Lyon: Internat. Agency for Research on Cancer, 9-152.

10. Švajdler, M., Mezencev, R., Ondič, O., Šašková, B., Mukenšnábl, P., Michal, M. (2018). P16 is a useful supplemental diagnostic marker of pulmonary small cell carcinoma in small biopsies and cytology specimens. Annals of Diagnostic Pathology, 33 , 23-29. doi: http://doi.org/10.1016/j.anndiagpath.2017.11.008

11. Dorantes-Heredia, R., Ruiz-Morales, J. M., Cano-García, F. (2016). Histopathological transformation to small-cell lung carcinoma in non-small cell lung carcinoma tumors. Translational Lung Cancer Research, 5 (4), 401-412. doi: http://doi.org/10.21037/tlcr.2016.07.10

12. Shuifang, C., Zeying, Z., Jianli, Z. (2019). The effects of the combination of imatinib and crizotinib on small cell lung cancer cells expressing c-Met and c-Kit. International Journal of Clinical and Experimental Medicine, 12 (5), 4870-4878. Available at: https://e-century.us/files/ijcem/12/5/ijcem0082071.pdf

13. Ramezani, M., Masnadjam, M., Azizi, A., Zavattaro, E. et. al. (2021). Evaluation of expression of c-Kit marker (CD117) in patients with squamous cell carcinoma (SCC) and basal cell carcinoma (BCC) of the skin. AIMS Molecular Science, 8 (1), 51-59. doi: http://doi.org/10.3934/molsci.2021004

14. Pelosi, G., Rindi, G., Travis, W. D., Papotti, M. (2014). Ki-67 Antigen in Lung Neuroendocrine Tumors: Unraveling a Role in Clinical Practice. Journal of Thoracic Oncology, 9 (3), 273-284. doi: http://doi.org/10.1097/jto.0000000000000092

15. Inoue, K., A. Fry, E. (2018). Aberrant expression of p16INK4a in human cancers - a new biomarker? Cancer Reports and Reviews, 2 (2). doi: http://doi.org/10.15761/crr.1000145

16. Pelosi, G., Masullo, M., Leon, M. E., Veronesi, G., Spaggiari, L., Pasini, F. et. al. (2004). CD117 immunoreactivity in high-grade neuroendocrine tumors of the lung: a comparative study of 39 large-cell neuroendocrine carcinomas and 27 surgically resected small-cell carcinomas. Virchows Archiv, 445 (5), 449-455. doi: http://doi.org/10.1007/s00428-004-1106-1

17. Jha, V., Sharma, P., Mandal, A. (2017). Utility of Cluster of Differentiation 5 and Cluster of Differentiation 117 Immunoprofile in Distinguishing Thymic Carcinoma from Pulmonary Squamous Cell Carcinoma: A Study on 1800 Nonsmall Cell Lung Cancer Cases. Indian Journal of Medical and Paediatric Oncology, 38 (4), 430-433. doi: http://doi.org/10.4103/ijmpo.ijmpo_148_16

18. Huang, Y., Chang, Y., Wu, C. (2019). EP1.09-10 A Diagnostic Pitfall in Posterior Mediastinal Tumor: Expression of CD117 in Atypical Ewing Sarcoma Masquerading as Classic Seminoma. Journal of Thoracic Oncology, 14 (10), S1001-S1002. doi: http://doi.org/10.1016/j.jtho.2019.08.2206

19. Ishibashi, N., Maebayashi, T., Aizawa, T., Sakaguchi, M., Nishimaki, H., Masuda, S. (2017). Correlation between the Ki67 proliferation index and response to radiation therapy in small cell lung cancer. Radiation Oncology, 12 (1). doi: http://doi.org/10.1186/s13014-016-0744-1

Received date 10.11.2021

Accepted date 16.12.2021

Published date 30.01.2022

Irina Yakovtsova*, Doctor of Medical Sciences, Professor, Head of Department, Department of Pathological Anatomy, Kharkiv Medical Academy of Postgraduate Education, Amosova str., 58, Kharkiv, Ukraine, 61176

Olexandr Yanchevskyi, Postgraduate Student, Department of Pathological Anatomy, Kharkiv Medical Academy of Postgraduate Education, Amosova str., 58, Kharkiv, Ukraine, 61176

Taisiia Chertenko, PhD, Assistant, Department of Pathological Anatomy, Kharkiv Medical Academy of Postgraduate Education, Amosova str., 58, Kharkiv, Ukraine, 61176

Andriy Kis, PhD, Associated Professor, Department of General and Clinical Pathology, V. N. Karazin Kharkiv National University, Svobody sq., 4, Kharkiv, Ukraine, 61022

Oliyinyk Andrii, PhD, Associated Professor, Department of Pathological Anatomy, Kharkiv Medical Academy of Postgraduate Education, Amosova str., 58, Kharkiv, Ukraine, 61176

*Corresponding author: Irina Yakovtsova, e-mail: docpathomorph@gmail.com 